\begin{tabular}{|c|l|}
\hline Title & Fabrication of Metal Embedded Nano-Cones for Single Quantum Dot Emission \\
\hline Author(s) & $\begin{array}{l}\text { Huh, Jae Hoon; Hermannstädter, Claus; A kahane, Kouichi; Sasakura, Hirotaka; Jahan, Nahid A .; Sasaki, Masahide; } \\
\text { Suemune, Ikuo }\end{array}$ \\
\hline Citation & $\begin{array}{l}\text { Japanese Journal of A pplied Physics, 50(6), 06GG02 } \\
\text { https://doi.org/10.1143/JJA.50.06GG02 }\end{array}$ \\
\hline Issue Date & 2011-06 \\
\hline Doc URL & http://hdl.handle.net/2115/52474 \\
\hline Rights & ○ 2011 The Japan Society of A pplied Physics \\
\hline Type & article (author version) \\
\hline File Information & MN10064.pdf \\
\hline
\end{tabular}

Instructions for use 


\title{
Fabrication of Metal Embedded Nano-Cones for Single Quantum Dot Emission
}

\author{
Jae-Hoon HUH, ${ }^{1,2}$ Claus HERMANNSTÄDTER, ${ }^{1}$ Kouichi AKAHANE, ${ }^{3}$ Hirotaka \\ SASAKURA, ${ }^{1,2}$ Nahid A. JAHAN, ${ }^{1}$ Masahide SASAKI, ${ }^{3}$ and Ikuo SUEMENE ${ }^{1,2}$ \\ ${ }^{1}$ Research Institute for Electronic Science, Hokkaido University, Sapporo 001-0021, Japan \\ ${ }^{2}$ Japan Science and Technology Corporation (CREST), Kawaguchi, Saitama 332-0012, Japan \\ ${ }^{3}$ National Institute of Information and Communications Technology, 4-2-1 Nukui-Kitamachi, Koganei, \\ Tokyo 184-8795, Japan \\ E-mail:hjaehoon@es.hokudai.ac.jp
}

\begin{abstract}
High efficiency in the extraction, transmission and detection of single and entangled photons is one of the most significant factors to provide general usage and to suppress the bit-error rate in optical communication networks. We propose and realize metal embedded nanostructures with quantum dots (QDs) as photon sources to meet these challenges on the emitter side. Advantages of the process are the ability of fast nanometer-scale fabrication and the high reproducibility and yield. Mesas with typical taper angles of $20-30^{\circ}$ and diameters between $100 \mathrm{~nm}$ and $1 \mu \mathrm{m}$ were produced; these nano-structures are referred to as "cones" in this work. The dependence of the taper angle on the composition of the etched cone material is discussed, focusing on the contribution of Indium. The nano-cones used as photon sources were embedded in highly reflective metal and turned upside down to provide efficient and partly directed photon extraction. We present the selection of $1.35 \mu \mathrm{m}$ photons emitted from a single QD as a result of embedding only a small number of dots in a nanometer sized cone.
\end{abstract}




\section{Introduction}

Quantum information processing and cryptography require high quality sources for single-photons and entangled photon pairs. Devices based on the luminescence from single self-assembled QDs are powerful candidates for such single and entangled photon sources ${ }^{1-8)}$. Due to the wide achievable spectral range from the ultraviolet to the near infrared, QDs can be used both in free space and in fiber-based communication networks at around 1.31 and $1.55 \mu \mathrm{m}$. The latter wavelength ranges are favorable for low loss transmission in networks of larger sizes, which is an essential requirement for efficient operation with a low bit-error rate.

To date, the main part of related reports is based on (In,Ga)As QDs grown on GaAs substrates where the wavelength range is limited to a maximum of $1.3 \mu \mathrm{m} .{ }^{9-11)}$ However, (In,Ga)As QDs grown on InP substrates with double-cap procedure have been reported as deterministic single photon sources (DSPs) at $1.55 \mu \mathrm{m} .{ }^{12-14)}$ Recently, K. Takemoto et al. reported the inclusion of QDs into $22^{\circ}$-tapered cones to avoid the backward propagation of emitted photons. They demonstrated a ten times enhanced photon extraction efficiency compared to bare self-assembled QDs.

In this paper, we report on our approach to realize a practical single photon source based on (In,Ga)As QDs, along with the ability of large scale fabrication. High density QDs (around $10^{11}$ per $\mathrm{cm}^{2}$ ) grown on InP substrates were tailored to emission wavelengths larger than $1.3 \mu \mathrm{m}$. Using these QD samples sub-micrometer sizes tapered cones were fabricated, which contain only a small number of QDs. As shown in the schematic figure 1 the tapered geometry was chosen in order to direct the emitted photons to the desired direction and to reduce losses inside the nanostructure. We present micro-photoluminescence $(\mu$-PL) spectra of nano-cones which feature 
atomic-like discrete sharp emission lines in the desired spectral range.

\section{Cone Fabrication}

The high density QD hetero-structures were grown by molecular beam epitaxy (MBE) lattice-matched to an $\operatorname{InP}(311) B$ substrate and consist of an $\operatorname{In}_{0.53} \mathrm{Al}_{0.22} \mathrm{Ga}_{0.25} \mathrm{As}$ buffer layer embedding 4 monolayers (ML) of InAs in the center. The cone structures were fabricated according to the six step process presented in figure 2. $\mathrm{A}_{\mathrm{SiO}_{2}}$ layer was deposited on the semiconductor surface by plasma chemical vapor deposition (PCVD) as etching mask for the $300 \mathrm{~nm}$ thick semiconductor layers because $\mathrm{SiO}_{2}$ exhibits a significantly lower etching rate than $\operatorname{In}_{0.53} \mathrm{Al}_{0.22} \mathrm{Ga}_{0.25} \mathrm{As}$. The negative tone resist Hydrogen Silsesquioxane (HSQ) was used for the electron-beam lithography (EBL) process of large scale patterns of differently sized cones. The patterns of $100 \mathrm{~nm}$ to 1

$\mu \mathrm{m}$ cones were written on the resist by $100 \mathrm{keV}$ EBL. The InAs/ $/ n_{0.53} \mathrm{Al}_{0.22} \mathrm{Ga}_{0.25} \mathrm{As}$ cones were dry etched using reactive ion etching (RIE) and inductively coupled plasma (ICP)-RIE with $\mathrm{CHF}_{3}$ and $\mathrm{Cl}_{2}$ etching gases, respectively. Resist patterning, $\mathrm{SiO}_{2}$ mask etching, semiconductor etching and mask removal were subsequently performed to fabricate the cone structures. A field-emission scanning electron microscope (SEM) equipped with dimension measurement tool (JEOL JSM-6700FT) was used to acquire SEM images under variable angles to quantify important parameters, such as, the cone dimensions and taper angles, as well as, the etching rates and the shape and remaining thickness of the mask.

We used a high density QD sample to ensure having dots embedded in cones of all fabricated sizes. Figure 3 (a) shows an AFM image of the as-grown uncapped 4 ML InAs QDs. The high dot density is due to the minimized lattice mismatch and thus strain 
of the InAs QDs grown on a lattice matched $\operatorname{In}_{0.53} \mathrm{Al}_{0.22} \mathrm{Ga}_{0.25} \mathrm{As}$ buffer (figure 3 (b)).

The respective low temperature PL spectrum shown in figure 3 (c) was obtained under non-resonant excitation. The PL of the $4 \mathrm{ML}$ QD ensemble is centered at a wavelength of $1.33 \mu \mathrm{m}$. The two further PL peaks at around 0.87 and $1.09 \mu \mathrm{m}$ originate from the InP substrate and the $\operatorname{In}_{0.53} \mathrm{Al}_{0.22} \mathrm{Ga}_{0.25} \mathrm{As}$ barrier, respectively.

In order to extract single QD luminescence from such a high density QD ensemble, the precise fabrication of small nanostructures with a good surface roughness is a crucial process. As shown in the SEM images in figure 4, we fabricated $25-28^{\circ}$ tapered cones, based on the given angle definition (figure $4(\mathrm{~d})$ ). We found that the mask etching rate and the high contrast EB writing are the most significant factors for the precise control of the cone size and shape. Furthermore, we carefully considered the impact of the remaining amount of $\mathrm{SiO}_{2}$ on the nano-fabrication. We found that the amount and shape of the remaining $\mathrm{SiO}_{2}$ and HSQ depends on the cone tip diameter and that it can be gradually reduced due to strong isotropic etching. The appearance of such formations is characteristic for $\mathrm{SiO}_{2}$ (and also for the HSQ resist) without an impact on the nanostructure size ${ }^{15)}$; however, this effect can contribute to enhance the taper angle of the nano-cones. As shown in figure 4 the taper angle is increasing with reducing the cone size and thus the $\mathrm{SiO}_{2}$ and HSQ amount on top. All these cones of different diameters were fabricated under the same etching condition at room temperature using $\mathrm{Cl}_{2}$ gas with a flow rate of $0.8 \mathrm{sccm}$ and a gas pressure of $0.03 \mathrm{~Pa}$; the ICP power was $150 \mathrm{~W}$ and the bias power $300 \mathrm{~W}$.

To address the question why the fabricated mesas are tapered and what causes and determines their taper angle, systematic etching studies were performed using compound semiconductors of different compositions. We found that usually GaAs 
mesas are almost straight with rather small taper angles between 0 and $4^{\circ}$ (figure 5 (a)). Mesas etched from an Aluminum containing compound, i.e., $\mathrm{Al}_{0.2} \mathrm{Ga}_{0.8} \mathrm{As}$, are no longer straight but exhibit slightly enhanced taper angles of $2-8^{\circ}$ (figure 5 (b)). In strong contrast to the two afore described cases the inclusion of Indium in the etched compound appears to be the key to produce strongly tapered mesas, i.e., cone structures. The fabricated $\operatorname{In}_{0.53} \mathrm{Al}_{0.22} \mathrm{Ga}_{0.25} \mathrm{As}$ mesas exhibit typical taper angles between 20 and $30^{\circ}$ (figure 5 (c)). The etching studies for the composition dependence of the taper angles were again all performed under the same etching conditions, as specified above. However, in the case of room temperature etching of the Indium-rich compound the substrate surface appears very rough because of the large amount of remaining Indium atoms. The Indium component is etched with $\mathrm{Cl}_{2}\left(\right.$ or $\left.\mathrm{CCl}_{4}\right)$ gas, but to obtain a smooth surface the substrate must be heated to above $200{ }^{\circ} \mathrm{C}$ to avoid Indium adsorption. ${ }^{16,17)}$ It has to be mentioned that the problem of enhanced surface roughness addressed before does not influence the quality of the nanostructures, the cone surface is clean and smooth as shown in figure 5 (c).

\section{Titanium-Embedded Cone}

\subsection{Fabrication}

Different sized cones were embedded in metal according to the three step process which is illustrated in the schematic figure 6. A $15 \mathrm{~nm}$ insulating $\mathrm{SiO}_{2}$ layer was deposited on the $\operatorname{In}_{0.53} \mathrm{Al}_{0.22} \mathrm{Ga}_{0.25} \mathrm{As}$ cones, followed by approximately $5 \mu \mathrm{m}$ of Titanium (figure 6 , step 1). Then the sample was turned around and mounted on a glass support substrate for the subsequent removal of the InP substrate by mechanical fine-polishing with a 0.3 
$\mu \mathrm{m}$ grit and selective chemical etching with a $\mathrm{HCl}+\mathrm{H}_{3} \mathrm{PO}_{4}(1: 1$ vol.) mixed solution (figure 6, steps 2 and 3).

Figure $7(a, b, e)$ shows a sequential illustration of the fabrication of an embedded nano-cone with the typical taper angle of $26^{\circ}$, as well as SEM images of the as-etched and embedded nano-cone. A few optically active QDs are located approximately 150 $\mathrm{nm}$ below the surface and at a sufficient distance to the semiconductor- $\mathrm{SiO}_{2}$-metal interface. The QDs located very close to this interface are optically inactive due to the damage occurring during the etching process and possible non-radiative recombination mechanisms mediated by the interface. Figure 7 (b) furthermore illustrates the relation between the different diameters of a nano-cone; for typical taper angles the top diameter of the embedded nano-cones is around $300 \mathrm{~nm}$ larger than the bottom diameter, the diameter of the QD plane is around $150 \mathrm{~nm}$ larger; for the displayed nano-cone these diameters are $100 \mathrm{~nm}$ (bottom), $256 \mathrm{~nm}$ (QD plane) and $412 \mathrm{~nm}$ (top). The optical microscope images in figures 7 (c, d) highlight the ability of fabricating large uniform areas of metal embedded nano-cones. They furthermore show the high yield of up to $100 \%$ for nano-cones of $\geq 100 \mathrm{~nm}$ bottom diameters and thus the good reproducibility of the process. The detailed SEM image, figure 7 (e), shows a single Titanium embedded nano-cone of the same dimensions as discussed in the schematic illustration in figure 7 (b); a nano-cone of this size was used for the PL spectroscopy discussed in the next paragraph.

\subsection{Micro-Photoluminescence}

Low temperature $\mu$-PL spectroscopy was performed under non-resonant excitation on a number of Ti-embedded nano-cones with sizes down to approximately $100 \mathrm{~nm}$ bottom 
diameter. The luminescence of these turned-around metal-embedded nano-cones was investigated at cryogenic temperatures using a double monochromator with exchangeable 300 and $1000 \mathrm{gr} / \mathrm{mm}$ gratings and an InGaAs-photodiode array detector. The low temperature $(\mathrm{T}=4 \mathrm{~K})$ QD s-shell emission of the $4 \mathrm{ML}$ sample is observed at wavelengths of 1.3-1.4 $\mu \mathrm{m}$. In figure 8 (a) a typical $\mu$-PL spectrum of a single nano-cone of around $100 \mathrm{~nm}$ bottom diameter is shown. Although the number of QDs in such a cone, estimated from the dot density and area, might be around 20-30, the main part of them is located close to the semiconductor- $\mathrm{SiO}_{2}$-metal interface and thus optically inactive as aforementioned (section 3.1 and figure 7 (b)). The displayed spectrum indicates that emission from only three to five optically active ("bright") QDs is visible, the remaining dots are rendered optically inactive ("dark") and are thus not of particular importance for the optical spectroscopy. The emission lines in the spectrum are clearly separable, such that, selecting the luminescence from only one QD is possible. The indicated spectral region around $1.36 \mu \mathrm{m}$ was examined in more detail as a function of excitation power (figure 8 (b)). A typical behavior for the PL of an uncharged QD could be observed with a neutral exciton recombination, X, that exhibits a linear dependence on excitation power and a biexciton recombination, $\mathrm{XX}$, that evolves with a super-linear dependence. The fit to the low power data exhibits a line width of the QD neutral exciton on the order of $0.1 \mathrm{meV}$. The appearance of such clearly isolated recombination lines from single QDs allows a potential usage as a source of single photons applying some additional spectral selection, e.g., by using suitable band-pass filters of less than $1 \mathrm{meV}$ band width to isolate individual PL peaks. Biexcion-exciton pairs are potential candidates for polarization entangled photons from the respective radiative cascade. ${ }^{6-8)}$ However, so far the assignment of PL lines due to 
the recombination of individual QD excitons and the preliminary classification of the underlying recombination processes has only been based on their line widths and spectral occurrence, as well as their excitation power dependence. A more detailed classification and prove of single QD and single photon emission will be performed in a separate experiment using photon statistics, i.e., second-order correlation spectroscopy.

\section{Conclusion}

We present an approach of fabricating precise nano-cone structures from high-density QD samples emitting at around $1.33 \mu \mathrm{m}$. The Indium content of the $\operatorname{In}_{0.53} \mathrm{Al}_{0.22} \mathrm{Ga}_{0.25} \mathrm{As}$ barrier material is the essential parameter to realize the $20-30^{\circ}$ tapered nanostructures. The cones were turned upside down after embedding them in metal and removing the substrate to ensure the best possible photon extraction. For the smallest realized Ti-embedded cones of about $100 \mathrm{~nm}$ bottom diameter emission from three to five optically active single dots was observed. The single-dot PL shows exciton-biexciton pairs and relatively small line widths of around $0.1 \mathrm{meV}$. The presented nano-cones are good candidates for the realization of long wavelength single photon emitters because of their small volume in line with a small number of contained optically active QDs and their efficient photon extraction. With the future option to shift the emission to $1.55 \mu \mathrm{m}$ by using larger QDs (6 ML InAs) or by higher temperature operation, these structures have the potential to be used as single photon sources in fiber-communication networks.

\section{Acknowledgments}

The authors acknowledge Dr. H. Kumano and Dr. S. Odashima for valuable comments 
and discussion and M. Wada for experimental assistance. This work was supported in part by the Grant-in-Aid for Scientific Research (A), No. 21246048 from the Ministry of Education, Science, Sports, and Culture, and by the Hokkaido Innovation through NanoTechnology Support (HINTS) for nanofabrication. C. H. acknowledges the Japan Society for the Promotion of Science (JSPS) for providing financial support in the form of a JSPS Research Fellowship for Young Scientists. 


\section{References}

1) P. Michler, A. Kiraz, C. Becher, W. V. Schoenfeld, P. M. Petroff, L. D. Zhang, E. Hu, and A. Imamoglu: Science 290 (2000) 2282.

2) Z. Yuan, B.E. Kardynal, R.M. Stevenson, A.J. Shields, C.J. Lobo, K. Cooper, N.S. Beattie, D.A. Ritchie, M. Pepper: Science 295 (2002) 102.

3) C. Santori, D. Fattal, J. Vukovic, G.S. Solomon, Y. Yamamoto: Nature 419 (2002) 594.

4) N. Gisin, G. Ribordy, W. Tittel, and H. Zbinden: Rev. Mod. Phys., 74 (2002) 145.

5) I. Friedler, C. Sauvan, J.P. Hugonin, P. Lalanne, J. Claudon, and J.M. Gérard: Optics Express, 17(4) (2009) 2095.

6) R. J. Young, R. M. Stevenson, P. Atkinson, K. Cooper, D. A. Ritchie, and A. J. Shields: New J. Phys. 8, (2006) 29.

7) N. Akopian, N. H. Lindner, E. Poem, Y. Berlatzky, J. Avron, D. Gershoni, B. D. Gerardot, and P. M. Petroff: Phys. Rev. Lett. 96, (2006) 130501.

8) R. Hafenbrak, S. M. Ulrich, P. Michler, L. Wang, A. Rastelli, and O. G. Schmidt: New J. Phys. 9, (2007) 315.

9) J.-Y. Marzin, J.-M. Gérard, A. Izraël, D. Barrier, and G. Bastard: Phys. Rev. Lett. 73, (1994) 716.

10) R. P. Mirin, J. P. Ibbetson, K. Nishia, A. C. Gossard and J. E. Bowers: Appl. Phys. Lett. 67, (1995) 3795.

11) K. Nishi, H. Saito, S. Sugou, and J.-S. Lee: Appl. Phys. Lett. 74, (1999) 1111.

12) K. Takemoto, Y. Sakuma, S. Hirose, T. Usuki, N. Yokoyama, T. Miyazawa, M. Takatsu, Y. Arakawa: Physica E 26 (2005) 185.

13) S. Fréchengues, N. Bertru, V. Drouot, B. Lambert, S. Robinet, S. Loualiche, D. 
Lacombe, A. Ponchet: Appl. Phys. Lett, 74, 22 (2000) 3356.

14) C. Paranthoen, N. Bertru, O. Dehaese, A. Le Corre, S. Loualiche, B. Lambert, G. Patriarche: Appl. Phys. Lett, 78, 12 (2001) 1751.

15) H. Norström, R. Buchta, F. Runovc, and P. Wiklund: Vacuum 32, 12 (1982) 737.

16) G. F. Doughty, S. Thomas, V. Law and C. D. W. Wilkinson: Vacuum 36, 11 (1986) 803.

17) V. M. Donnelly, D. L. Flamm, and D. E. Lbbotson: J. Vac. Sci. Technol.A, 1(2) (1983) 626. 


\section{Figure captions}

Fig. 1: Schematic illustration of light extraction from an (a) un-tapered mesa and (b) tapered mesa (cone) surrounded by highly reflective metal.

Fig. 2: Cone structure fabrication process: (Step 1) mask formation of $\mathrm{SiO}_{2}$ and $\mathrm{HSQ}$ resist, (Step 2) EB writing of different sized cones, (Step 3) resist patterning by development, (Step 4) $\mathrm{SiO}_{2}$ etching by RIE, (Step 5) $\mathrm{In}_{0.53} \mathrm{Al}_{0.22} \mathrm{Ga}_{0.25} \mathrm{As}$ etching by ICP-RIE, (Step 6) fluoric acid treatment for mask removal.

Fig. 3: (a) AFM images of uncapped InAs/ $\operatorname{In}_{0.53} \mathrm{Al}_{0.22} \mathrm{Ga}_{0.25} \mathrm{As}$ QD samples with a nominal InAs deposition thickness of $4 \mathrm{ML}$, (b) schematic illustration of the unstructured samples, and (c) low temperature PL (non-resonant excitation at $532 \mathrm{~nm}$ ) with the QD ensemble PL peaked at $1.33 \mu \mathrm{m}$.

Fig. 4: SEM images of tapered cone structures with different tip diameters and the remaining $\mathrm{SiO}_{2}$ mask; (a) $98 \mathrm{~nm}$, (b) $398 \mathrm{~nm}$, (c) $864 \mathrm{~nm}$; and (d) schematic illustration of the taper angle definition and measurement. All cones were obtained under the same etching condition $\left(\mathrm{Cl}_{2}\right.$ gas with flow rate $0.8 \mathrm{sccm}$, gas pressure $0.03 \mathrm{~Pa}$, ICP power 150 $\mathrm{W}$ and bias power $300 \mathrm{~W}$, room temperature).

Fig. 5: SEM images of mesas/cones of different semiconductor compounds fabricated under the same etching condition $\left(\mathrm{Cl}_{2}\right.$ gas with flow rate $0.8 \mathrm{sccm}$, gas pressure $0.03 \mathrm{~Pa}$, ICP power $150 \mathrm{~W}$ and bias power $300 \mathrm{~W}$, room temperature); (a) GaAs 0-4 ${ }^{\circ}$ tapered, (b) $\mathrm{Al}_{0.2} \mathrm{Ga}_{0.8} \mathrm{As} 2-8^{\circ}$ tapered, and (c) $\operatorname{In}_{0.53} \mathrm{Al}_{0.22} \mathrm{Ga}_{0.25} \mathrm{As} 20-30^{\circ}$ tapered. 
Fig. 6: Ti-embedded cone fabrication and substrate removal process: (Step 1) $\mathrm{Ti}$ deposition, (Step 2) mechanical fine-polishing, and (Step 3) selective chemical etching.

Fig. 7: (a) SEM image of a $26^{\circ}$-tapered nano-cone with approximately $100 \mathrm{~nm}$ tip diameter. (b) Schematic illustration of the turned-around Ti-embedded nano-cone containing a few QDs with the following diameters: bottom $100 \mathrm{~nm}$, center (QD plane) $256 \mathrm{~nm}$ and top $412 \mathrm{~nm}$. The optical images (c, d) show areas of fabricated nano-cones arranged in a pattern of $20 \mu \mathrm{m}$ cone-to-cone separation, the SEM image (e) shows a single Ti-embedded nano-cone of $412 \mathrm{~nm}$ top diameter.

Fig. 8: $\mu$-PL spectra obtained from a nano-cone (4 ML QDs) of the dimensions described in figure 7 (b, e) at a temperature of $4 \mathrm{~K}$ under non-resonant $\mathrm{cw}$ excitation; (a) shows a wide-range PL spectrum containing emission lines of a few individual QDs and (b) shows the excitation power dependent PL of a narrower spectral region (dashed lines in (a)) exhibiting a single QD with two discrete PL lines which are assigned to as the neutral exciton $(\mathrm{X})$ and the biexciton $(\mathrm{XX})$ recombination, respectively. 
Figure 1 (1 col.)

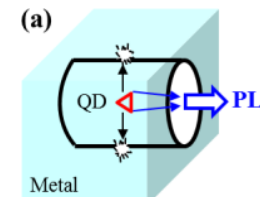

(b)

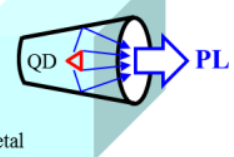


Figure 2 (2 col.)

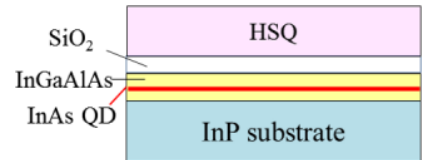

(Step 1)
$\Rightarrow$

InP substrate

(Step 2)

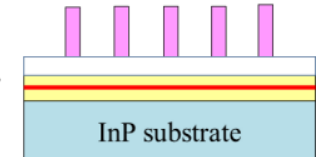

(Step 3)

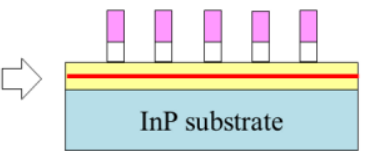

(Step 4)

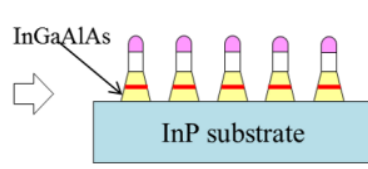

(Step 5)

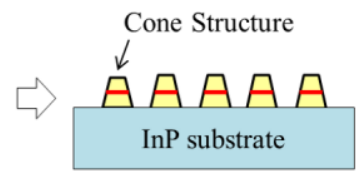

(Step 6) 
Figure 3 (2 col.)
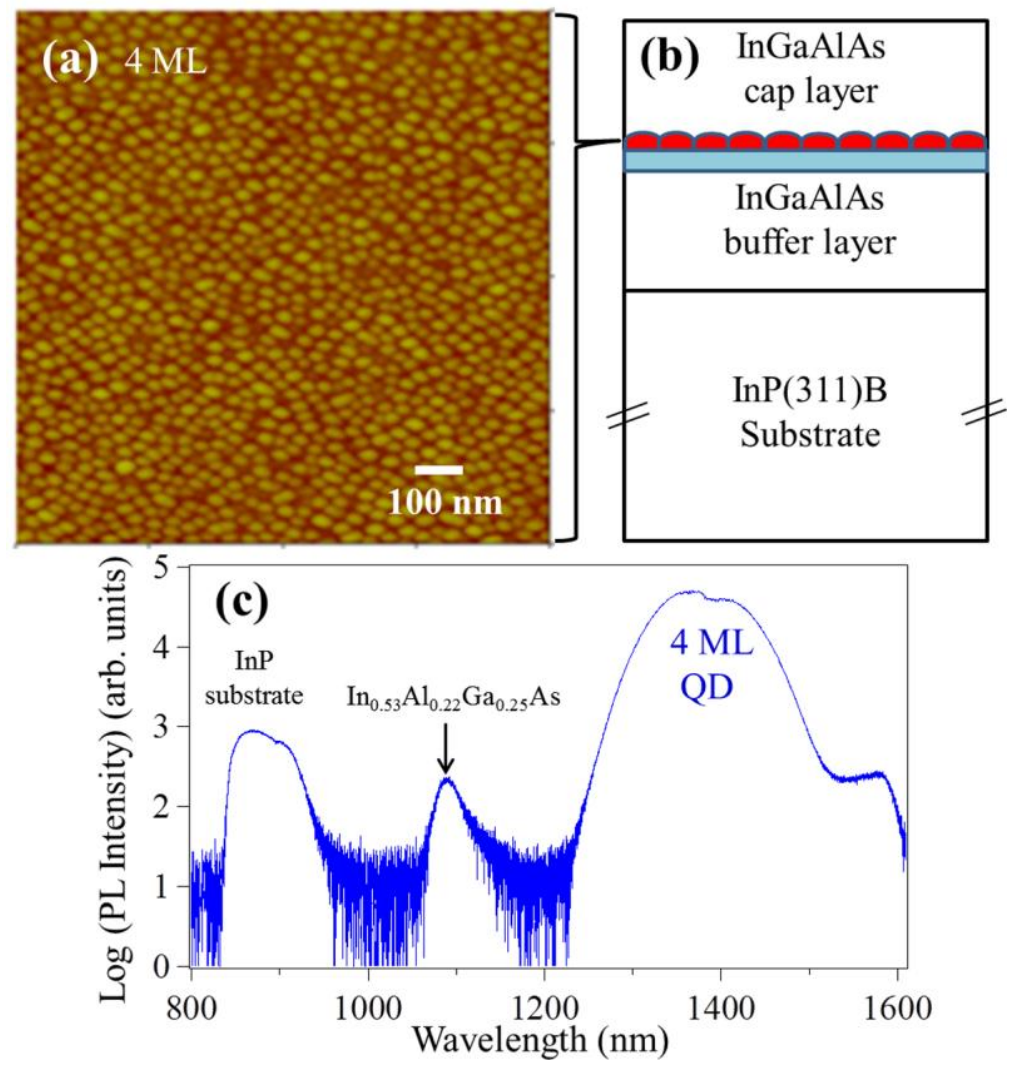
Figure 4 (1 col.)
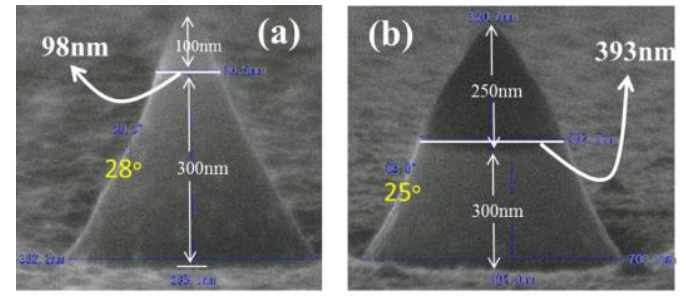

(c)

(d)

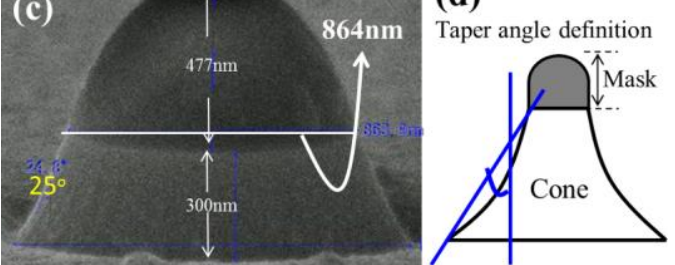


Figure 5 (1 col.)
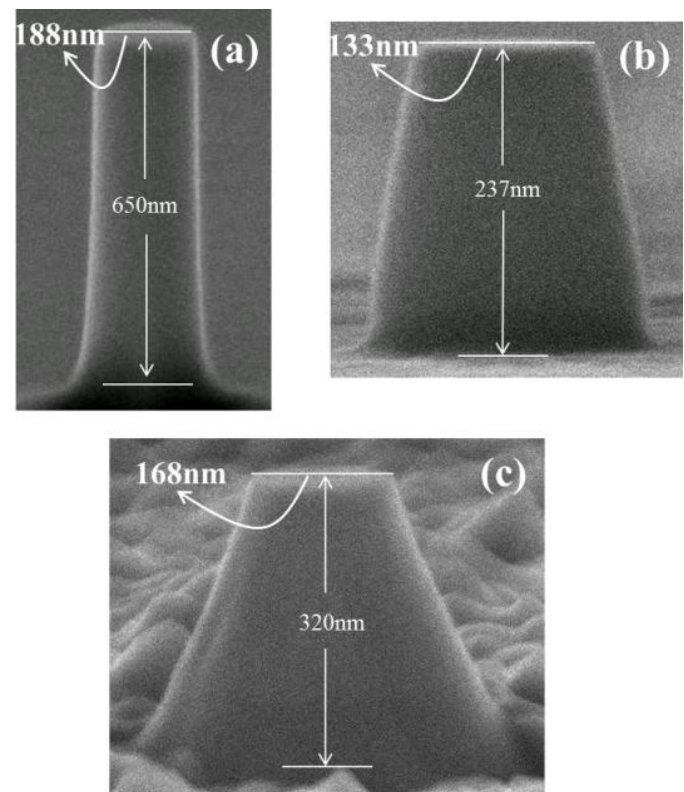
Figure 6 (2 col.)

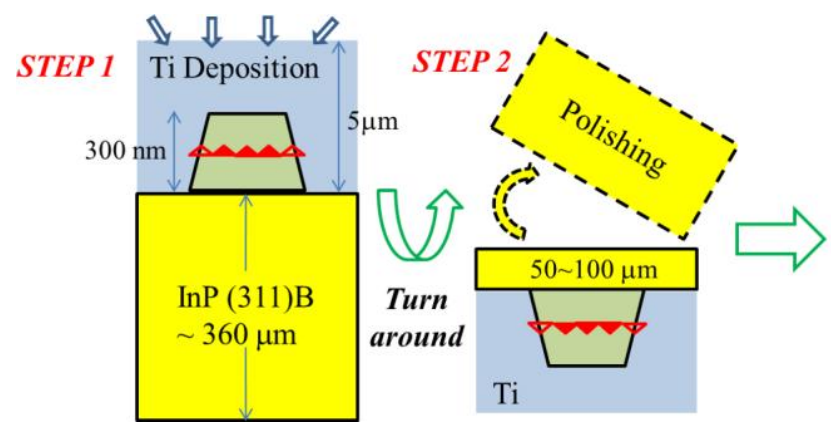

STEP 3

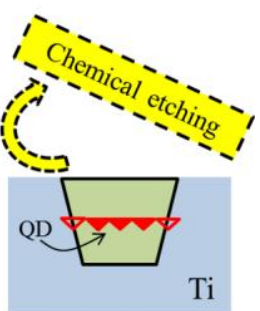


Figure 7 ( 2 col.)

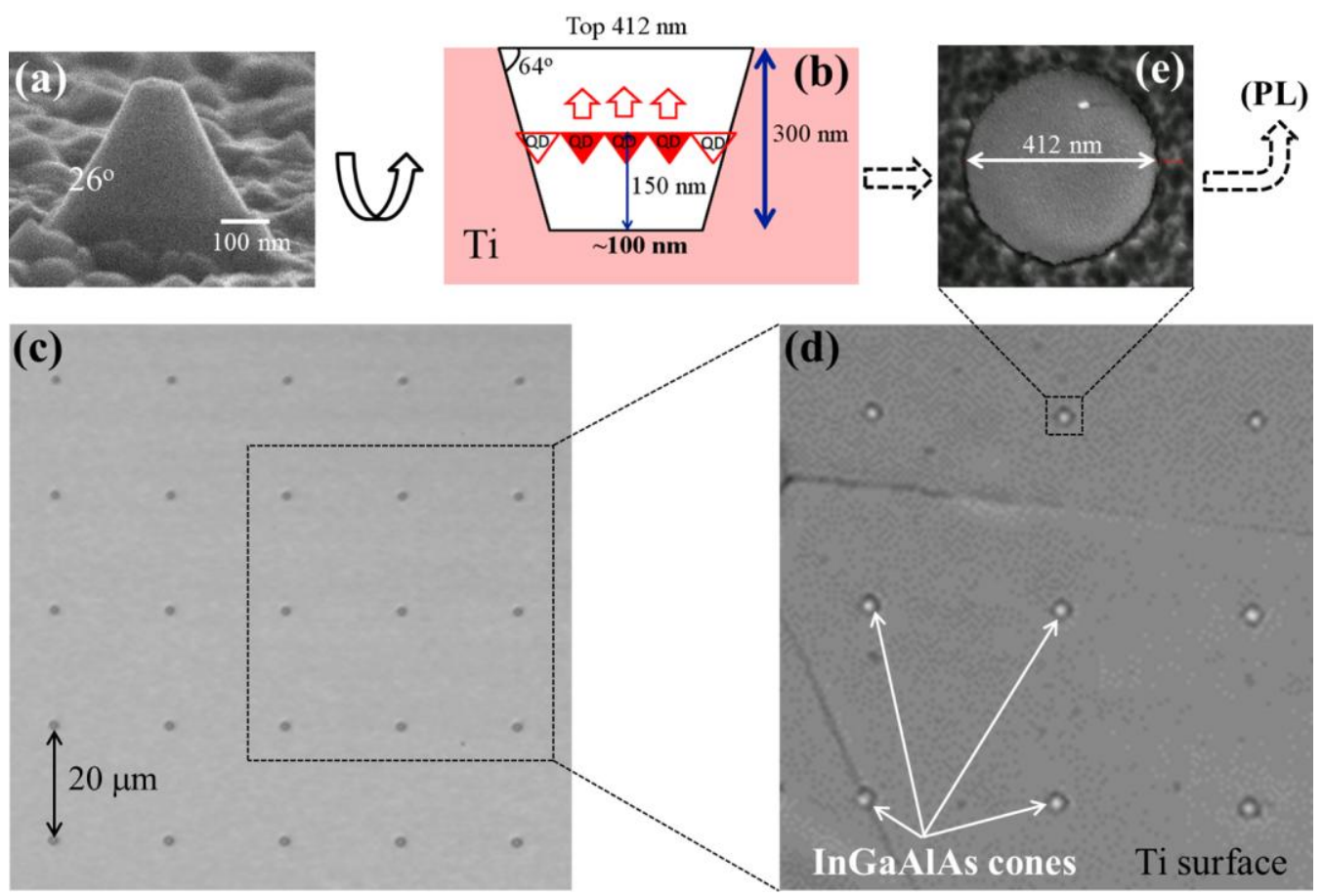


Figure 8 ( 1 col.)
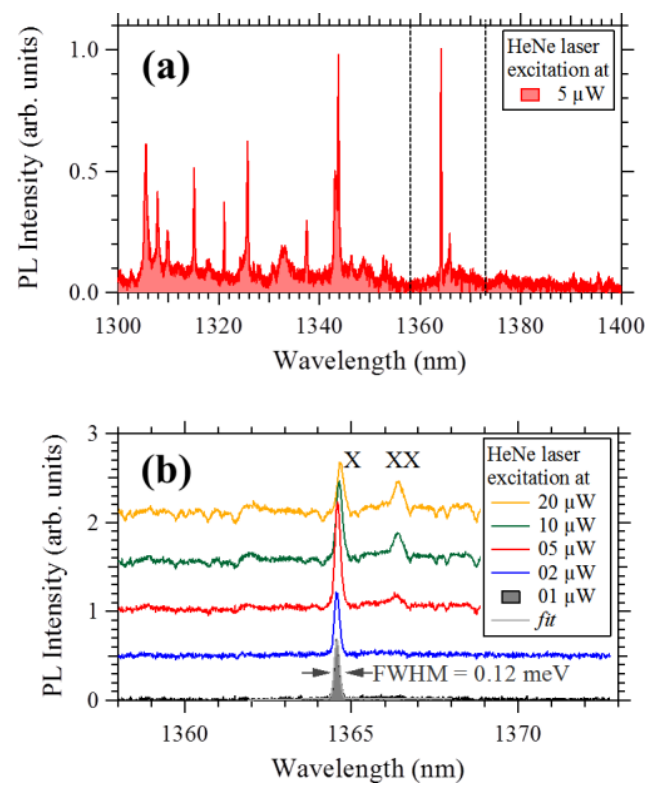